\title{
La Base de Datos de Ciudades Históricas de Andalucía: un instrumento para la Ordenación del Territorio y la protección del Patrimonio Histórico
}

\author{
José Manuel Díaz Iglesias \\ Centro de Documentación. IAPH. \\ Consejería de Cultura \\ Juan Luis Díaz Quidiello \\ Servicio de Planeamiento Regional. \\ Consejería de Obras Públicas y Transportes \\ Pilar Hurtado Gallardo \\ Servicio de Protección del Patrimonio Histórico. \\ Consejería de Cultura \\ Valle Muñoz Cruz \\ Centro de Documentación. IAPH. \\ Consejería de Cultura \\ $\mathrm{M}^{\text {a }}$ Angeles Muñoz Rubio \\ Servicio de Planeamiento Urbanistico. \\ Consejería de Obras Públicas y Transportes
}

\section{Resumen}

Se presenta una aplicación informática para la gestión y el conocimiento de las Ciudades Históricas andaluzas desarrollada conjuntamente por técnicos de la Consejería de Obras Públicas y Transporte y de la Consejería de Cultura de la Junta de Andalucía en el marco del proyecto europeo de cooperación "Redes de Ciudades Históricas de Influencia Islámica en el Sur de la Península Ibérica y Norte de Marruecos". El objetivo de esta iniciativa es contar con un instrumento que resulte útil en las actuaciones sobre ordenación del territorio y gestión del Patrimonio Histórico. En líneas generales se expone el alcance, el ámbito, los contenidos, la estructura informática de la aplicación y las perspectivas de continuidad del proyecto.

\section{Palabras clave}

Ciudades Históricas / Conjuntos Históricos / Patrimonio Urbano / Patrimonio Histórico / Gestión / Ordenación del territorio / Protección / Andalucía / Bases de Datos / Sistemas de Información.

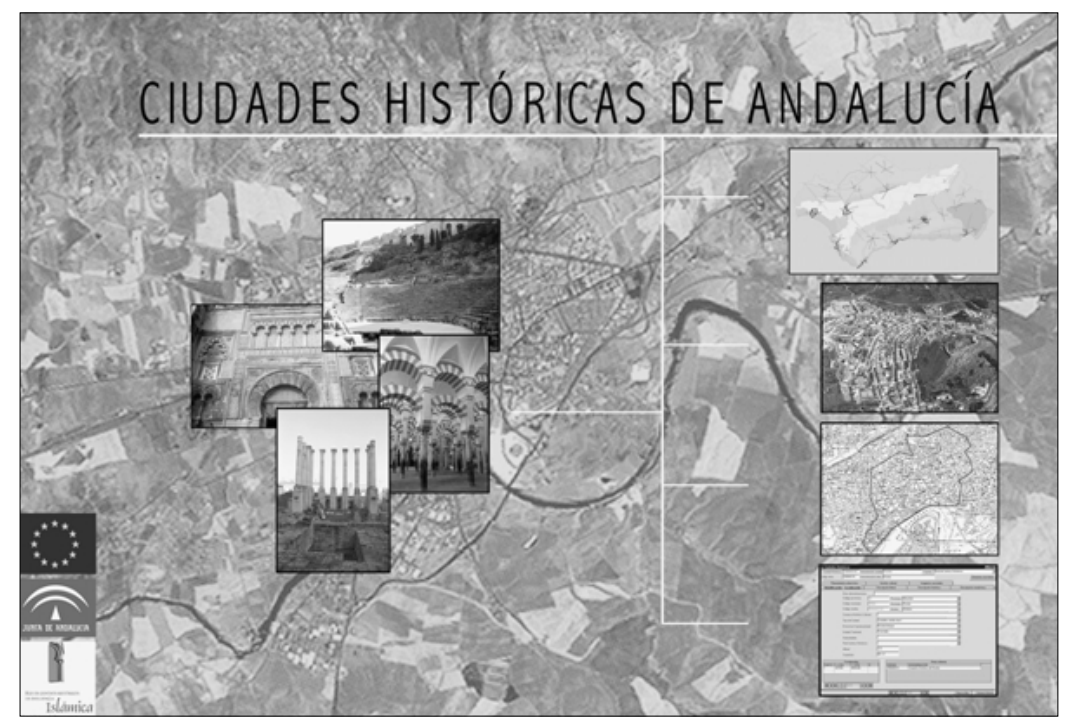

La creación de una base de datos sobre Ciudades Históricas de Andalucía es una iniciativa conjunta de las Consejerías de Obras Públicas y Transportes y de Cultura de la Junta de Andalucía, que tiene su origen en el Proyecto "Redes de Ciudades Históricas de Influencia Islámica en el Sur de la Península Ibérica y Norte de Marruecos" incluido en la Acción Piloto de cooperación entre España, Portugal y Marruecos (artículo 10 del FEDER I).

Los antecedentes de esta Acción Piloto hay que buscarlos en las diversas líneas de trabajo que desde la Unión Europea procuran crear un marco de cooperación (político, económico, técnico, cultural...) entre las regiones de la Península lbérica y entre éstas y los países y regiones del Mediterráneo, y en concreto con Marruecos.

El primer paso de cooperación entre los socios españoles (Andalucía y Extremadura) y portugueses (Alentejo y Algarve) se dio a través de la iniciativa Comunitaria Interreg I (1990-1993) con un Programa Operativo que abarcaba la zona transfronteriza entre los dos países. La continuidad de este Programa se garantizó con el Interreg II (1994-1999) que además de la cooperación entre España y Portugal, incorpora la posibilidad de desarrollar acciones de cooperación de Andalucía con Marruecos.

Por otro lado las Consejerías de Obras Públicas y Transportes y de Cultura, desde la década de los no- 
venta, vienen desarrollando de forma coordinada diversas líneas de actuación en los Centros Históricos. Como resultado se publicó en 1994 la Orden conjunta de ambas Consejerías en la que se aprueba el Programa Regional de Planeamiento en Centros Históricos donde se recoge la necesaria coordinación de las administraciones urbanísticas y culturales en el ámbito de la ordenación del territorio y el Patrimonio Histórico. Para ello propone programas y líneas de actuación que promulguen la cooperación municipal y el apoyo a la formulación del planeamiento.

\section{Alcance y significado}

Dentro del Proyecto de Redes de Ciudades Históricas de Influencia Islámica, la Acción I denominada "Base de Datos Multimedia sobre el Patrimonio Cultural Común" planteaba dos objetivos:

- La creación y mantenimiento de una Base de Datos sobre la Red de Centros Históricos y los elementos patrimoniales comunes de origen o influencia islámica.

- La producción de un CD-ROM sobre la misma temática.

Inicialmente se trataba de dos acciones concebidas como dos fases de un único proceso, siendo el CDROM un producto de difusión de los resultados obtenidos en la base de datos. Varias razones, sin embargo, han aconsejado desarrollarlas de forma independiente. Por una parte, los plazos fijados no permitían esperar al diseño y la carga de una base de datos para elaborar posteriormente el CD-ROM. Por otra, las diferentes situaciones en las que se encontraban los sistemas de información de cada uno de los socios (Junta de Andalucía, Junta de Extremadura, CCR del Algarve, Campo Arqueológico de Mértola y Reino de Marruecos) hacían inviable construir una base de datos común para todo el ámbito de la acción, sin realizar previamente un importante esfuerzo de homogeneización. Sí se han abordado en común, en cambio, las tareas específicas necesarias para editar el CD-ROM, pero éste se ha realizado a partir de la elaboración de materiales confeccionados expresamente para su producción.

En definitiva, la acción originaria se ha desdoblado en dos actuaciones independientes con ámbitos, contenidos y finalidades distintas. Una de ellas ha sido la creación de una Base de Datos sobre Ciudades Históricas limitada exclusivamente a la Comunidad Autónoma Andaluza, cuya temática se ha ampliado a todo el patrimonio urbano regional con independencia de su relación con el periodo islámico y cuya finalidad principal es apoyar la gestión que de este patrimonio tiene encomendada la Junta de Andalucía.

Esta última decisión, la de crear una base de datos sobre ciudades entre las dos Consejerías y de ampliar su ámbito a todas las ciudades históricas andaluzas, con independencia de cualquier limitación cronológica, temática o tipológica, tiene una especial trascendencia, entre otras cuestiones, por tres motivos.

En primer lugar, porque constituye una iniciativa pionera de cooperación entre Departamentos dentro

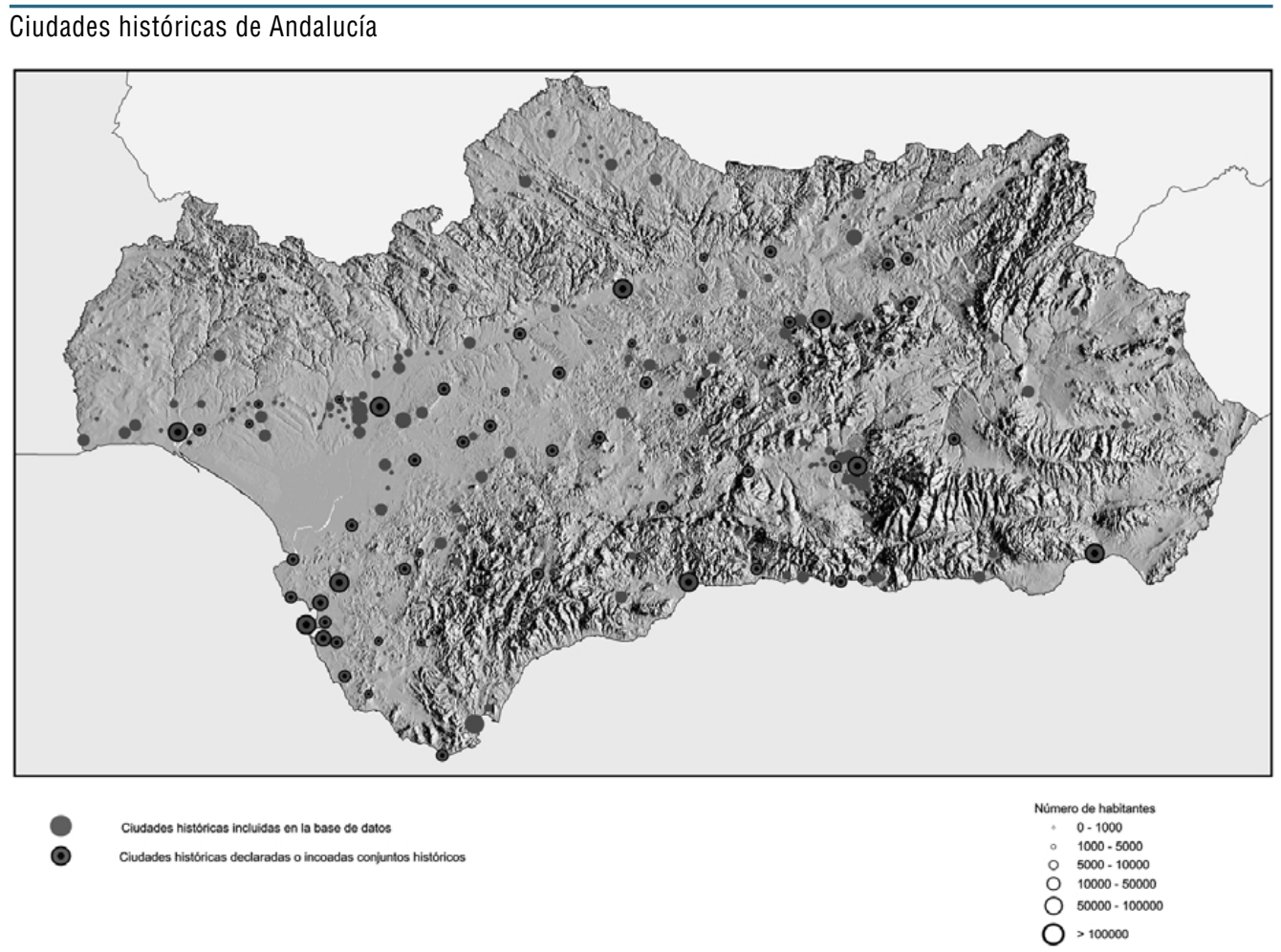


de la Junta de Andalucía y más concretamente entre sus respectivos sistemas de información: el Sistema de Información Territorial (SIT), de la Consejería de Obras Públicas y Transportes, y el Sistema de Información del Patrimonio Histórico de Andalucía (SIPHA), perteneciente a la Consejería de Cultura. La trascendencia de la iniciativa estriba en el hecho de que no se trata sólo de intercambiar determinadas informaciones -algo relativamente habitual -, sino de construir una única Base de Datos a partir de las aportaciones de las dos Consejerías y gestionarla posteriormente de forma igualmente conjunta. La colaboración iniciada entre ambos sistemas debe interpretarse, en consecuencia, como una apuesta por superar la generalizada tendencia a compartimentar artificialmente las actuaciones y abrir, así, el camino a futuras colaboraciones.

En segundo lugar, porque la Base de Datos, que se crea, implica reconocer que la política sobre el Patrimonio Cultural y, en particular la relacionada con la Ciudad Histórica, debe tener una naturaleza integral y que solamente será verdaderamente eficaz en la medida que en ella participen todos los órganos de la administración con responsabilidades en la materia. En definitiva, que no puede disociarse la protección del patrimonio cultural de la ordenación del territorio y el urbanismo. Es por ello que el diseño de la Base de Datos no debe entenderse en ningún caso como la simple yuxtaposición de contenidos de responsabilidad exclusiva de cada una de las Consejerías.

$Y$ en tercer lugar, porque se trata de formar una base de información que cubre todo el patrimonio urbano regional, entendido éste en un sentido amplio, de acuerdo con los planteamientos formulados en el Plan General de Bienes Culturales y en las Bases y Estrategias del Plan de Ordenación del Territorio de Andalucía. El proyecto contempla no sólo a los Conjuntos Históricos identificados como tales a los efectos de la legislación sobre Patrimonio Histórico (Ley | 6/85 del Patrimonio Histórico Español y Ley |/9 | del Patrimonio Histórico de Andalucía), sino también al resto de los asentamientos urbanos que son una expresión significativa de las relaciones históricas de la sociedad andaluza con su territorio con independencia del valor monumental que pueda reconocerse a cada uno de estos núcleos. El ámbito del proyecto abarca, por esta razón, a todos los centros urbanos que se han considerado relevantes para las políticas de protección y puesta en valor del patrimonio urbano y territorial que son responsabilidad de los dos Departamentos de la Junta de Andalucía implicados en el programa.

La Base de Datos sobre Ciudades Históricas debe entenderse, por tanto, en este marco de superación de una concepción del patrimonio urbano que quedaba reducido sólo a determinados elementos singulares y desvinculada de contextos territoriales y sociales más amplios. El objetivo último no es otro que contar con un instrumento de una política más general de protección y puesta en valor de las ciudades históricas ya no sólo como valiosos objetos patrimo- niales aislados, sino como bienes territoriales de alta potencialidad para un desarrollo socioeconómico y territorial armónico.

Por varias razones la iniciativa está llamada a ser especialmente útil para la gestión autonómica, en primer lugar, para identificar los recursos patrimoniales con los que pueden contar las distintas políticas regionales de desarrollo y ordenación del territorio y, en segundo lugar, para poder realizar una evaluación de las actuaciones que en materia de protección y puesta en valor de las ciudades históricas puedan ponerse en marcha.

\section{El diseño}

La Base de Datos ahora elaborada ha partido para su diseño de la experiencia acumulada en la materia por las dos Consejerías implicadas. En concreto, el Centro de Documentación del IAPH venía ya trabajando desde 1997 en el desarrollo de una base de datos de Conjuntos Históricos de Andalucía ${ }^{2}$, y posteriormente durante el 2000 se implementó una nueva aplicación que amplió el ámbito de estudio a las Ciudades Históricas Andaluzas ${ }^{3}$, haciendo posible la realización de análisis de áreas urbanas e incluyendo un módulo de navegación cartográfica ${ }^{4}$.

A partir de estos antecedentes el proyecto de "Red de Centros Históricos de influencia islámica" ha permitido el diseño y desarrollo informático de una nueva aplicación que incorpora cambios y avances significativos. A continuación se hace una relación de los más importantes:

- Se ha establecido el centro histórico como la unidad básica de información. Frente a otras opciones (el término municipal, el núcleo de población o, en su caso, el Conjunto Histórico) se ha optado por delimitar dentro de cada núcleo de población el espacio que se ha considerado que constituye la ciudad histórica y a él se refiere la información incorporada a la base de datos. La delimitación ha sido el resultado de un análisis previo morfológico de cada uno de los núcleos seleccionados.

- Se han incorporado variaciones cualitativas en los módulos de información de la anterior base de datos que se comentan en el apartado de Estructura.

- Se ha ampliado el módulo de elementos de interés que en la anterior aplicación incluía sólo unos datos muy básicos de los inmuebles de la ciudad, y se ha permitido el acceso a toda la información del Patrimonio Inmueble de Andalucía disponible en el SIPHA: elementos arqueológicos, arquitectónicos y etnológicos 5 .

- Desde un punto de vista funcional la novedad más interesante de la aplicación es que se ha incorporado un módulo de consulta común para el Territorio, Ciudades y Patrimonio Inmueble. El sistema 
permite la realización de búsquedas sencillas y un módulo de consultas avanzadas a través de la combinación de operadores booleanos. También se han incorporado distintos modelos de informes de salida tanto para ciudades, áreas urbanas y elementos de interés.

- Por otro lado, se ha modificado la estructura para ampliar y mejorar la información territorial y gráfica. El criterio seguido ha sido el de sustituir siempre que fuese posible la información alfanumérica por informaciones gráficas o cartográficas.

- Con respecto al visualizador cartográfico, aunque en líneas generales se mantiene la estructura de la anterior aplicación, el nuevo aporta otras posibilidades, entre las que destacan: la edición de coberturas para modificar los elementos de la misma, la mejora de las opciones de impresión y exportación, y, por último, la incorporación de nuevas coberturas.

\section{El ámbito}

El primer paso del proyecto ha consistido en la delimitación de su propio ámbito, esto es, la identificación de las ciudades que deben tener la consideración de "Ciudades Históricas" de interés tanto para la protección del patrimonio cultural, como para las distintas políticas territoriales. En esta labor se ha contado con los trabajos y proyectos realizados con anterioridad por las dos Consejerías y, en concreto con:

- Las ciudades que formaban parte de la anterior base de datos "Ciudades Históricas de Andalucía" del SIPHA de la Consejería de Cultura. En la misma se incluía información sobre los Conjuntos Históricos de Andalucía.

- Las ciudades comprendidas en el estudio "Análisis Urbanístico de los Conjuntos Históricos de Andalucía: ciudades medias y pequeñas" realizado por la Consejería de Obras Públicas y Transporte, que abarca a 122 núcleos, muchos de ellos coincidentes con los incorporados a la base de datos del SIPHA.

- Los núcleos que han sido objeto del proyecto de investigación "Tipología de Redes de Centros Históricos Andaluces" realizado en el marco del Programa Regional de Planeamiento en Centros Históricos, y que ha abarcado un número sensiblemente superior de asentamientos. En total el estudio recogió más de 400 núcleos, seleccionados por formar algún tipo de red territorial histórica, que en muchos casos no coinciden con las ciudades contempladas anteriormente. Por una parte, quedaron excluidas no sólo las grandes ciudades - como ya sucedía en el "Análisis Urbanístico de los Conjuntos Históricos de Andalucía: ciudades medias y pequeñas"- si no también núcleos con cierto grado de interés, que, sin embargo, no fue posible integrarlos en ninguna de las redes definidas.
Por el contrario, sí se incluyeron muchas ciudades y asentamientos, que respondían a tipologías habitualmente no consideradas (los poblados de colonización recientes o los poblados mineros, por ejemplo), o por tratarse de núcleos que sin disfrutar de grandes singularidades patrimoniales, tienen, en cambio, una importancia relevante como partes de redes históricas de asentamientos.

El conjunto de estas tres selecciones previas dio como resultado una primera identificación de ciudades históricas que ha sido sometida, a su vez, a varios dictámenes de expertos en patrimonio histórico y ordenación del territorio, lo que ha permitido perfilar una selección en torno a 500 ciudades y núcleos de población con la que inicialmente se ha puesto en marcha la base de datos. No se trata, en cualquier caso, de una selección cerrada y está previsto seguir contrastando su idoneidad a lo largo de todo el proceso de carga de información y de utilización de la misma por los distintos departamentos de la Administración autonómica.

\section{La carga de nueva información}

La Base de Datos de Ciudades Históricas incluye la información proporcionada por los trabajos arriba mencionados y la carga de nueva información. Aunque no puede darse por concluido el proceso, -en parte porque la propia finalidad de la base implica una constante actualización, y, en parte, porque los objetivos planteados superan con creces los límites temporales y presupuestarios impuestos por la propia acción-, los avances realizados han sido considerables y permiten disponer de un volumen importante de información útil y suficientemente contrastada. Los trabajos más relevantes llevados a cabo han sido:

- Se han sustituido los campos alfanuméricos que originariamente se destinaban a encuadrar geográficamente y administrativamente cada una de las ciudades por mapas de encuadre territorial que permiten visualizar directamente las relaciones más básicas de cada centro histórico con su entorno territorial.

- Se han incorporado las delimitaciones de las ciudades históricas sobre la cartografía a escala 1:10.000 de los actuales asentamientos de población.

- Se ha incluido una serie de planos y fotografías históricas de cada una de las ciudades, que comprende reproducciones de la denominada primera serie del Instituto Geográfico Nacional (mapas de finales del siglo XIX y principios del siglo XX), de fotografías aéreas de 1956 (el conocido como "vuelo americano") y de fotografías aéreas de 1996. La serie hace posible reconstruir la evolución seguida por la ciudad histórica y sus relaciones con su entorno durante el último siglo en tres momentos especialmente significativos para la evolución de las ciudades andaluzas. 
- Se ha realizado el escaneado de los documentos (tanto escritos como gráficos) publicados en los Boletines Oficiales de los Conjuntos Históricos y de los Entornos de los Monumentos tanto declarados como incoados BIC o inscritos en el Catálogo General del Patrimonio Histórico. Cuando no consta delimitación gráfica publicada se han escaneado las delimitaciones existentes en los expedientes administrativos. También se han interpretado estas mismas delimitaciones gráficas a una cartografía a escala 1:2.000 ó 1/5.000 dependiendo de la disponibilidad del Instituto de Cartografía de Andalucía. La incorporación de este trabajo permitirá resolver algunas de las confusiones que planteaban las interpretaciones de las delimitaciones realizadas con anterioridad.

- Se han revisado las descripciones literales contenidas en la primera base de datos y se han realizado nuevas descripciones para las ciudades y núcleos incorporados en esta nueva fase. La información literal hace referencia al emplazamiento, la topografía y geografía del mismo, la estructura urbana, la tipología edificatoria y las transformaciones recientes.

- Se ha realizado una clasificación tipológica del conjunto de ciudades siguiendo la metodología ya utilizada en el estudio "Análisis urbanístico de los centros históricos de Andalucía. Ciudades pequeñas y medianas", que contempla la siguiente clasificación: tipo de emplazamiento, forma del centro, tipo de estructura urbana, posición relativa y origen.

\section{La estructura}

La aplicación de Ciudades Históricas Andaluzas se está desarrollando en Visual-Basic sobre Oracle. Oracle es un gestor de bases de datos de gran potencia que facilita la gestión de aplicaciones en red. El área del visualizador está desarrollada en Visual Basic, usando la librería de controles de objetos geográficos de ESRI MapObjects LT 2.0.

El Sistema se estructura en dos áreas: el visualizador cartográfico y las bases de datos alfanuméricas Ciudades Históricas y Patrimonio Inmueble ${ }^{6}$. Ambas áreas están vinculadas de tal forma que es posible entrar directamente a las bases de datos o a través del visualizador. Asimismo la base de datos de Ciudades Históricas está relacionada con la del Patrimonio Inmueble a través del módulo de Elementos de Interés.

Funcionalmente, desde el visualizador cartográfico es posible realizar una serie de tareas de forma independiente a las bases de datos o conectar con estas a través del módulo de consultas.

De este modo el menú de herramientas del visualizador permite:

- La navegación y localización cartográfica a través de las diferentes coberturas.
- La gestión y edición de las mismas.

- Mostrar la información asociada a cada elemento.

- Realizar consultas desde el visualizador seleccionando a través de los diferentes campos de cada cobertura la información que se desea visualizar.

- Imprimir la vista seleccionada o exportarla a un formato gráfico ráster convencional.

La base de datos de Ciudades Históricas se estructura funcionalmente en un módulo de Altas/Modificaciones y otro de Consultas/Informes. A través del Módulo de ALTAS/MODIFICACIONES puede realizarse la carga de información desde los siguientes módulos de análisis: Identificación-Localización, Imágenes asociadas, Elementos de Interés, Descripción literal, Descripción histórica, Descripción estadística, Planeamiento urbanístico, Gestión Cultural y Documentación (Información Bibliográfica, Información Documental e Información Gráfica)

A través del módulo de Identificación-localización se ha puesto en relación cada una de las ciudades tanto con sus referentes administrativos (núcleo de población, término municipal, etc.), como con los ámbitos derivados de la planificación territorial y, en concreto con las propuestas contenidas en el Modelo Territorial de Andalucía de las Bases y Estrategias del Plan de Ordenación del Territorio de Andalucía (Unidades Territoriales, Tipos de ciudad y Estructuras Urbanas Supramunicipales) (Figura 3).

A través de este módulo puede accederse a los Elementos de Interés de la Ciudad Histórica o del área seleccionada.

El módulo de Imágenes asociadas permite la visualización de toda la documentación gráica asociada a la ciudad: mapas, planos, y fotografías (Figura 4)

El módulo de Descripción literal permite definir las características territoriales y urbanas generales del área de estudio. Primero a través de una aproxima-

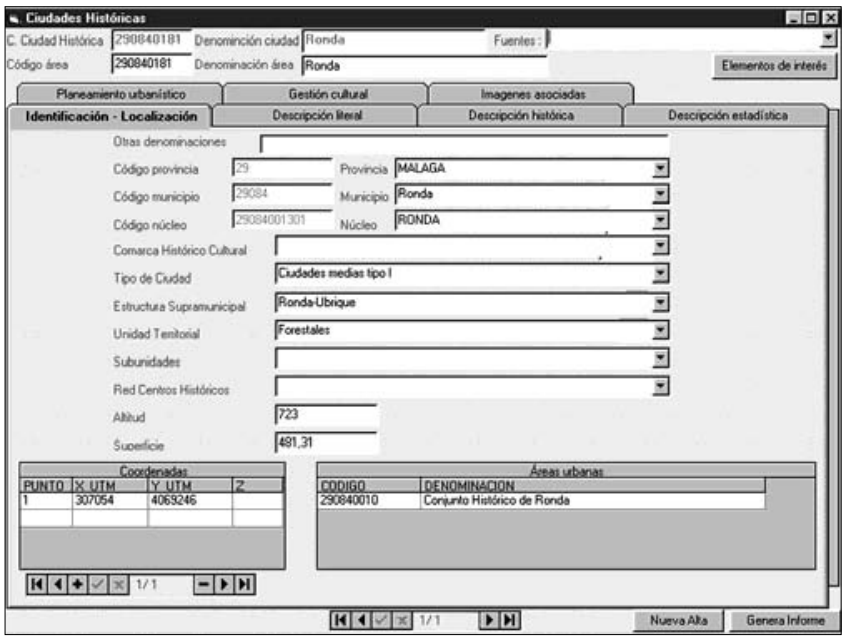




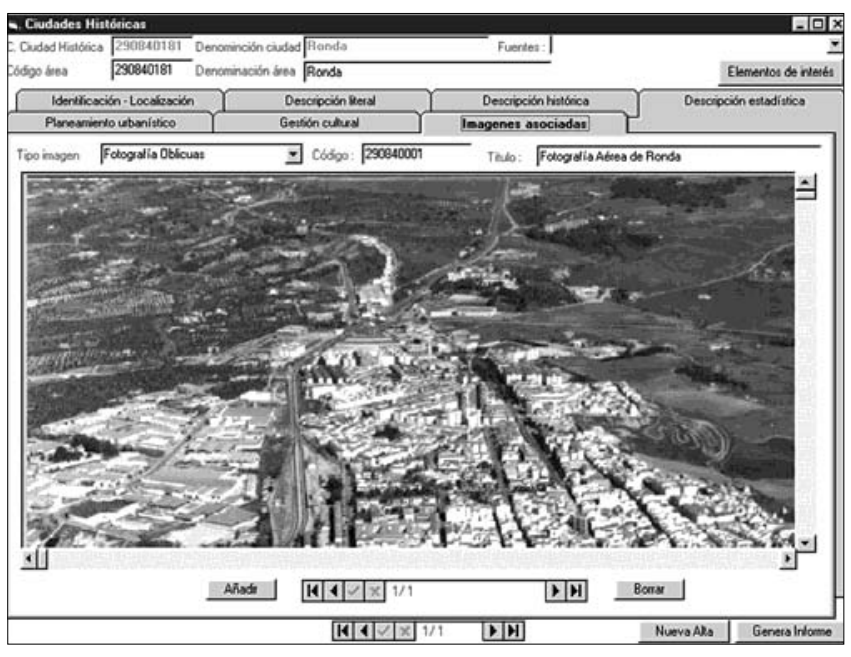

Fig. 4

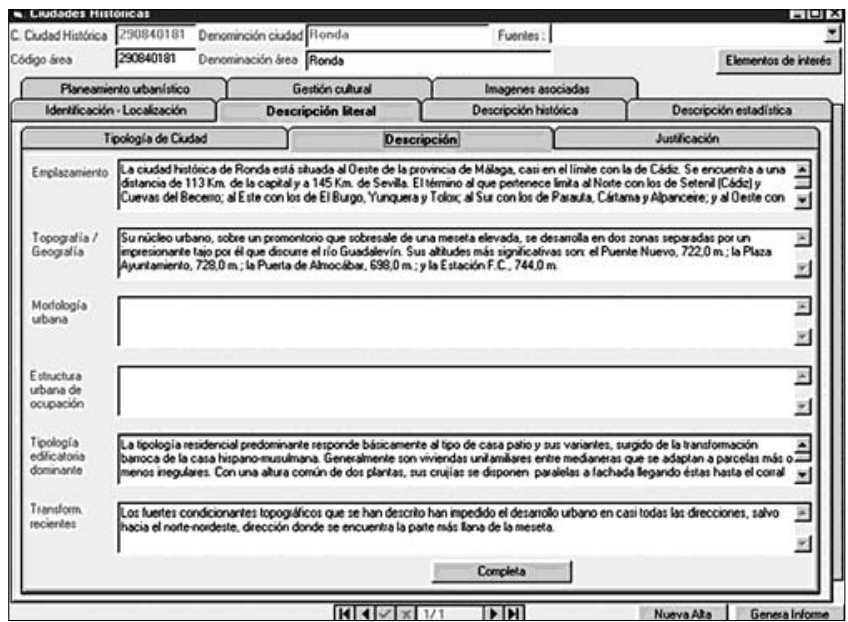

Fig. 5

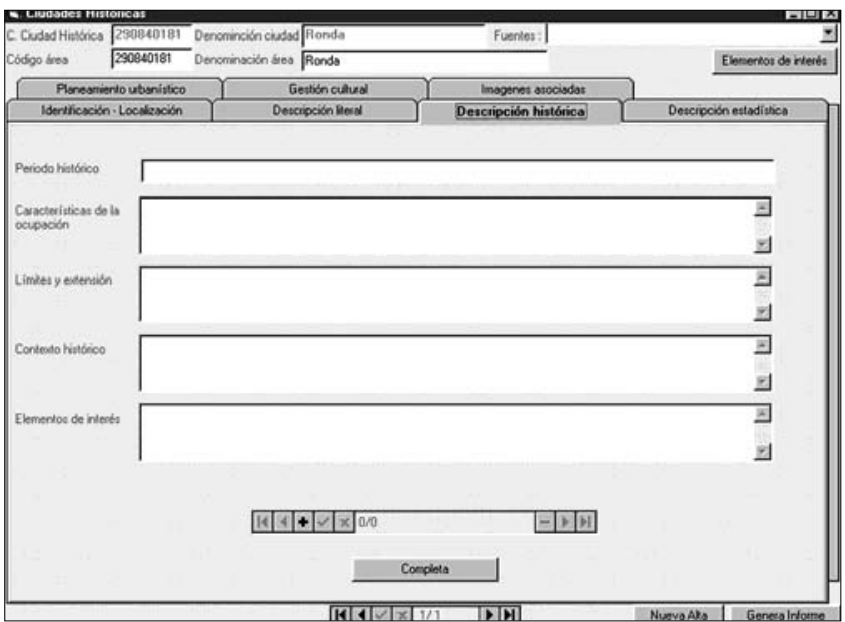

cion tipológica atendiendo a cinco criterios de clasificación: según el tipo de emplazamiento, la forma del centro histórico, la estructura urbana, la posición relativa centro/núcleo y el origen. Y a continuación a través de un campo articulado a su vez en una serie de apartados que caracterizan de forma general el área urbana: emplazamiento, topografía, estructura urbana de ocupación, etc. El módulo termina con un campo para la justificación de la inclusión, en donde se exponen las razones por las que la ciudad en cuestión o el área urbana identificada en ella se consideran Ciudad Histórica o parte de la misma (Figura 5).
El módulo de Descripción histórica tiene por objeto la definición de las características históricas más significativas de la ciudad o el área urbana seleccionada. Los campos de este módulo son: características de la ocupación, límites y extensión, contexto histórico y elementos de interés. La estructura de campos de este módulo podrá repetirse para cada uno de los periodos históricos analizados ${ }^{7}$ (Figura 6).

El módulo de Descripción estadística se ha simplificado bastante en relación a la anterior aplicación, debido fundamentalmente a la imposibilidad de conseguir determinados datos y a la falta de actualización de otros. El módulo ha quedado dividido en dos apartados: demografía y vivienda. El apartado de demografía recoge datos relativos al número de habitantes, superficie en $\mathrm{Km}^{2}$, densidad, unidades familiares y $n^{\circ}$ de miembros. Respecto a la vivienda se incluyen campos relativos al total de viviendas, viviendas principales, secundarias y desocupadas, alojamientos y viviendas colectivas (Figura 7).

Con respecto al Planeamiento urbanístico, se han ampliado y modificado los campos con la finalidad de poder realizar un seguimiento más específico de sus contenidos de protección. El módulo está estructurado en dos grandes apartados, el primero presenta los datos de identificación del planeamiento urbanístico que afecta a la ciudad o área urbana analizada (clase, figura, tipo, denominación, datos de publicación, convalidación por Cultura y su disposición). En el segundo apartado, más amplio que el anterior, se incluyen campos referentes a la protección individualizada desde el planeamiento urbanístico (si hay o no catálogo, datos sobre éste, etc.). Se recoge también una información general sobre los inmuebles protegidos por el catálogo y algunos campos relacionados con la protección arqueológica (Figura 8).

El módulo de Gestión Cultural está estructurado en tres apartados, el primero es Protección, que presenta información relacionada con la protección de los Conjuntos Históricos. El segundo apartado de Intervención/conservación incluye información de las actuaciones sobre inmuebles realizadas en el área urbana considerada. Por último, el tercer apartado de Intervención arqueológica recoge información sobre las intervenciones arqueológicas realizadas en el área urbana analizada. (Figura 9).

Con respecto al módulo de Documentación, éste a su vez se subdivide en los módulos de Información $\mathrm{Bi}$ bliográfica, Documental y Gráfica que recogen unos datos básicos de la documentación existente sobre la ciudad o el área de la ciudad objeto de estudio ${ }^{8}$.

Por último, a través del botón de Elementos de Interés se accede en primer lugar a un listado básico de todos los elementos incluidos en el área seleccionada, a partir del cual es posible consultar toda la información existente sobre el mismo. En concreto sus datos de Identificación-descripción (Figura 10), Localización, Delimitación Entorno, Protección, Planeamiento, Conservación-Intervención y Documentación. 
El Módulo de CONSULTAS/INFORMES de la aplicación presenta una pantalla común para Ciudades y Patrimonio Inmueble que permite realizar una consulta de tipo territorial o ir acotando la consulta a través de un encuadre territorial. Los campos de búsqueda de esta pantalla son unidades territoriales, subunidades, estructura supramunicipal, red de centros, comarca histórico-cultural y a continuación una serie de áreas administrativas: provincia, término municipal y núcleo de población. El programa permite ir seleccionando los términos de búsqueda a través de listas asociadas o introducir directamente el término de búsqueda. Una vez realizado el encuadre territorial se puede optar por realizar una consulta más específica de Ciudades o de Patrimonio Inmueble (Figura II).

El módulo de consulta de Ciudades Históricas permite realizar búsquedas a través de la selección de áreas urbanas: Ciudad histórica, Conjunto Histórico y Zona Arqueológica (urbana). A continuación es posible seguir acotando la búsqueda y para ello la pantalla se ha estructurado en cuatro apartados que a su vez se subdividen en campos. Los apartados son Tipología de ciudad, Estadística, Planeamiento y Protección (Figura 12). Por último desde el módulo de consulta de Ciudades Históricas es posible seguir concretando la consulta sobre elementos de interés de una determinada área urbana.

Una vez elaborada la ecuación de búsqueda el resultado se puede visualizar a través de un listado, así mismo es posible ver los datos de los registros seleccionados y elaborar informes, que podrán grabarse o imprimirse. Se han diseñado distintos modelos de informes básicos y completos para Ciudades, Conjuntos/Zonas Arqueológicas, Áreas urbanas y Elementos de interés.

A través del módulo de Administración tendrán que definirse las funciones de los distintos usuarios del Sistema: carga, consulta, validación, etc. Con respecto a la función de validación de la información, determinados usuarios estarán autorizados a validar la información cargada desde los distintos puntos de la red, de tal manera que ninguna información formará parte del banco de datos sin ser revisada previamente por dichos técnicos.

\section{Perspectivas de futuro}

La Base de Datos de Ciudades Históricas no es un proyecto cerrado y debe concebirse como un sistema vivo que deberá alimentarse desde las áreas de gestión y también a través de proyectos específicos. Será necesario todavía un periodo de prueba para detectar problemas de diseño, deficiencias en el funcionamiento, etc. que deberán cubrirse en fases posteriores. La continuidad del proyecto permitirá, en este sentido, solventar algunos vacíos y deficiencias en el diseño de la aplicación que sólo se harán patentes con su utilización.
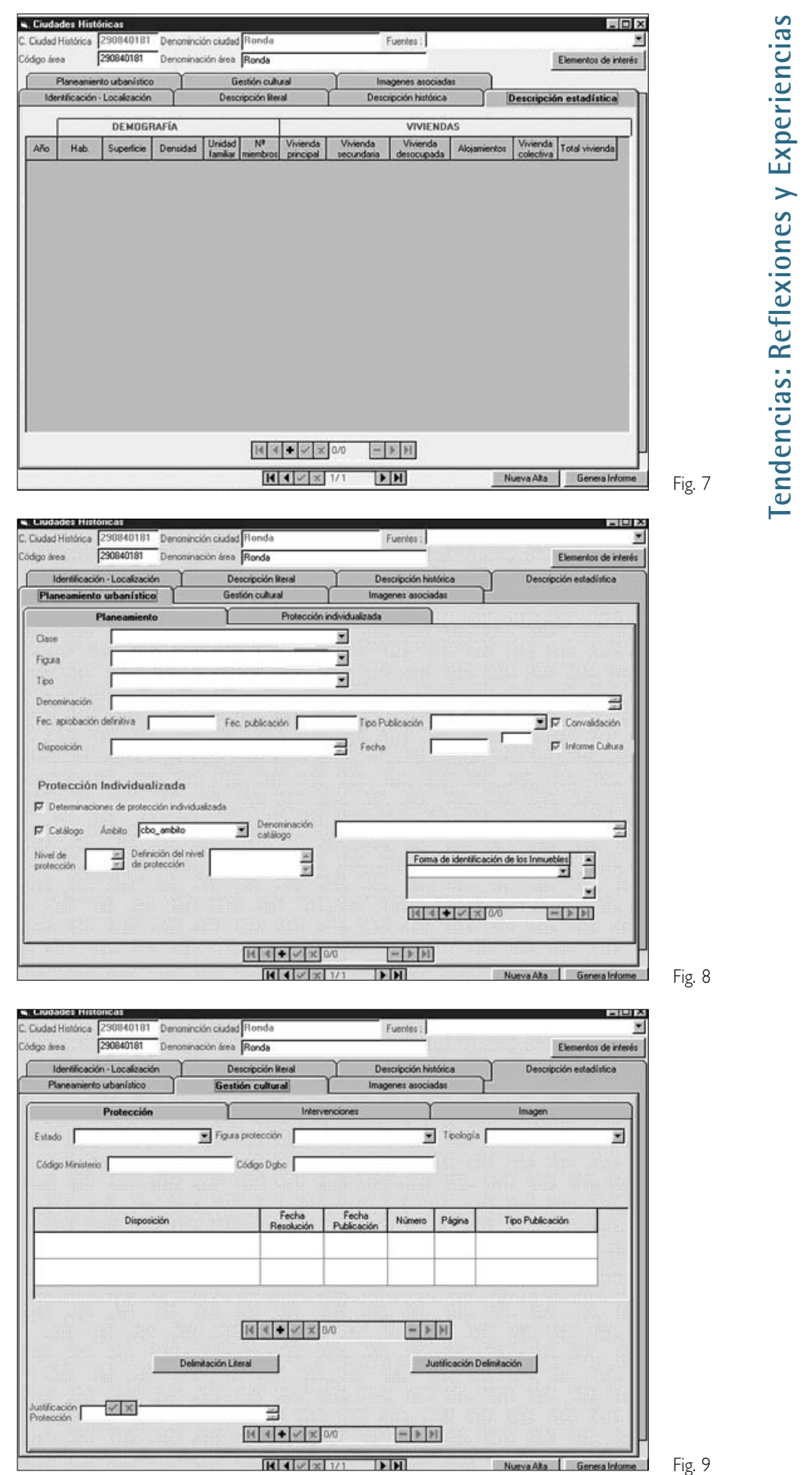

La Base de Datos concebida como un instrumento destinado a enriquecer el conocimiento sobre las Ciudades Históricas de Andalucía, se planteó para uso en gran parte interno de la propia Administración Autonómica, para mejorar la calidad en la gestión del Patrimonio, pero también al servicio de otras administraciones, instituciones y profesionales relacionados con su tutela. Por lo tanto deberá contemplarse, en el futuro, la participación de otros agentes con competencias en la ordenación del territorio y el Patrimonio Histórico. Así mismo deberá proponerse el diseño y desarrollo de productos de 


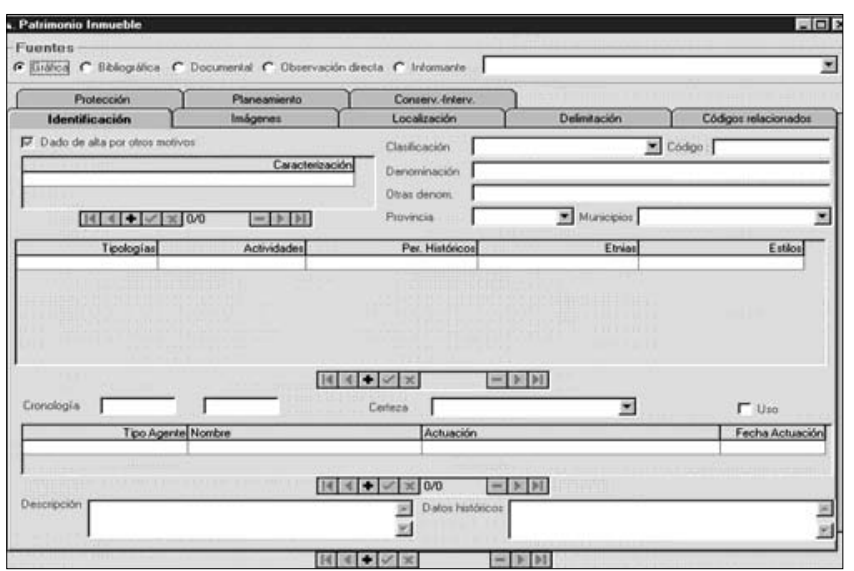

Fig. 10

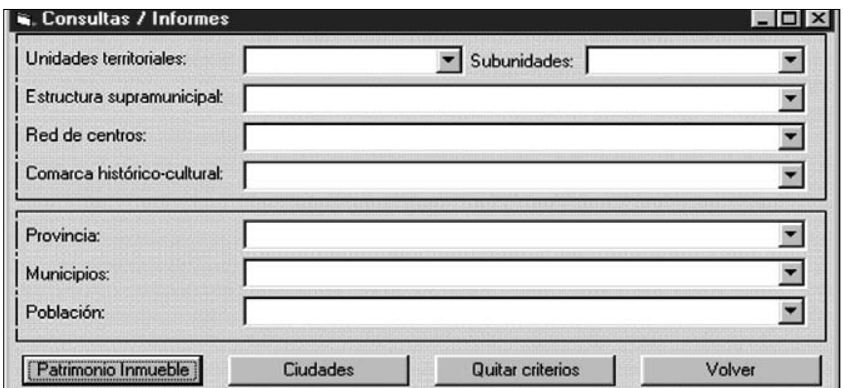

Fig. II

Fig. 12 difusión para diferentes tipos de usuarios. En este sentido está prevista, para la siguiente fase del proyecto, el desarrollo de una aplicación en Internet que ponga a disposición de cualquier usuario información sobre las Ciudades Históricas de Andalucía.

Para garantizar la continuidad del proyecto se cuenta con varios instrumentos. Uno de ellos es el que proporciona el Programa Regional de Planeamiento en Centros Históricos de Andalucía que prevé la realización de actuaciones conjuntas de las dos Consejerías relativas a la recopilación de información sobre las ciudades históricas. En concreto una de las líneas de actuación previstas es la de fomento de la investigación -en 1996 se realiza la primera convocatoria de Ayudas a la Investigación y en Octubre del 2000 la segunda-

Otra posibilidad de futuro podría plantearse a través de la continuidad de los proyectos europeos. De hecho, actualmente se ha realizado una propuesta para el Proyecto Heritage II, programa europeo al que se han presentado básicamente los mismos socios del proyecto FEDER en el que se encuadra la Base de Datos de Ciudades Históricas. Este nuevo proyecto incluye como objetivo principal la creación de una base de datos en el que el nivel de las regiones participantes alcancen un nivel homogéneo de gestión y dinamización del Patrimonio Cultural de origen común.

Tras la experiencia adquirida se ha constatado las grandes posibilidades de cooperación en cuanto a recopilación y tratamiento de la información y su posterior difusión. Por lo tanto, lo que se pretende es, por un lado, compatibilizar las Bases de Datos de cada región, y por otro, ampliar y diversificar los contenidos para continuar con la alimentación y tratamiento de la información. Para ello se ha planteado la creación de oficinas técnicas en cada región que se encargarían, coordinadamente, de estas tareas.
I. Este proyecto comenzó en 2000 y su periodo de vigencia termina el 3 I de diciembre de 200 I. Incluye, junto a la creación de una Base de Datos Multimedia, otras actuaciones, tales como: estudios, investigación e intercambios de experiencias, planes de interpretación, centros de Interpretación, itinerarios, etc., todo ello complementado con actos de dinamización y divulgación de las actividades y resultados. Sobre su contenido se puede consultar el artículo que sobre el mismo aparece en este Boletín.

2. La base se nutrió de información facilitada por la Consejería de Obras Públicas y Transporte, la Consejería de Cultura, el Instituto Cartográfico de Andalucía y el Instituto de Estadística de Andalucía. PICO VALIMAÑA, R.; PÉREZ HUMANES, M. Patrimonio Arquitectónico y Urbanístico en Andalucía: hacia la configuración de un sistema de información territorial. En Boletín IAPH, Año VI, N²2, Marzo, 1998. P. 106-109.

3. TAPIA MARTÍN, C. La base de datos de Ciudades Históricas Andaluzas. En Boletín IAPH, Año VIII, № 3I, Junio, 2000, p. |88-19|.

4. El Área de Arqueología del Centro de Documentación ya había desarrollado la aplicación ARQUEOSMapa que incluía gran parte de las funciones del visualizador que se incorporan ahora a la aplicación de Ciudades Históricas Andaluzas. FERNÁNDEZ CACHO,S; MONDÉJAR FDEZ. DE QUINCOCES, P.; DÍAZ IGLESIAS, J.M. Arqueos: un modelo andaluz de gestión de la información arqueológica. En Boletín IAPH, Año VIII, No 30, marzo, 2000, pp. I43- I49.

5. Sobre la base de datos del Patrimonio Inmueble de Andalucía se puede consultar el artículo que sobre este tema aparece en este Boletín.

6. Sobre la base de datos de Patrimonio Inmueble se puede consultar el artículo que aparece en este Boletín

7. Tanto el módulo de descripción literal como el de descripción histórica están estructurados en apartados o campos que faciliten la redacción por parte del usuario de la aplicación, pero la aplicación permite la visualización por campos o por texto completo

8. Estos módulos son una versión reducida de las bases de datos documentales del Centro de Documentación del IAPH. 